\title{
Recovering Variable Names for Minified Code with Usage Contexts
}

\author{
Hieu Tran*, Ngoc Tran*, Son Nguyen*, Hoan Nguyen ${ }^{\dagger}$, and Tien N. Nguyen* \\ ${ }^{*}$ Computer Science Department, The University of Texas at Dallas, USA, \\ Email: \{trunghieu.tran,nmt140230,sonnguyen,tien.n.nguyen\}@utdallas.edu \\ ${ }^{\dagger}$ Computer Science Department, Iowa State University, USA, Email: hoan@iastate.edu
}

\begin{abstract}
To avoid the exposure of original source code in a Web application, the variable names in JS code deployed in the wild are often replaced by short, meaningless names, thus making the code extremely difficult to manually understand and analysis. This paper presents JSNEAT, an information retrieval (IR)-based approach to recover the variable names in minified JS code. JSNEAT follows a data-driven approach to recover names by searching for them in a large corpus of open-source JS code. We use three types of contexts to match a variable in given minified code against the corpus including the context of the properties and roles of the variable, the context of that variable and relations with other variables under recovery, and the context of the task of the function to which the variable contributes. We performed several empirical experiments to evaluate JSNEAT on the dataset of more than 322K JS files with $1 \mathrm{M}$ functions, and $3.5 \mathrm{M}$ variables with $176 \mathrm{~K}$ unique variable names. We found that JSNEAT achieves a high accuracy of $69.1 \%$, which is the relative improvements of $66.1 \%$ and $43 \%$ over two state-of-theart approaches JSNice and JSNaughty, respectively. The time to recover for a file or a variable with JSNEAT is twice as fast as with JSNice and $4 x$ as fast as with JNaughty, respectively.
\end{abstract}

Keywords-Minified JS Code, Variable Name Recovery, Naturalness of Code, Usage Contexts.

\section{INTRODUCTION}

Software developers have to spend a significant portion of their efforts in comprehending the code. An important aspect of program understanding is the names of the identifiers used in the source code [2]. Meaningful identifiers help developers tremendously in quickly grasping the essence of the code. Thus, naming conventions are strongly emphasized on prescribing how to choose meaningful variable names in coding standards [1]. These principles also apply to Web development.

Web technologies and programming languages require the exposure of source code to Web browsers in the client side to be executed there. To avoid such exposure, the source code such as JavaScript (JS) files are often obfuscated in which the variable names are minified, i.e., the variable names are replaced with short, opaque, and meaningless names. The intention has two folds. First, it makes the JS files smaller and thus is quickly loaded for better performance. Second, minification diminishes code readability to hide business logics from the readers, while maintaining the program semantics.

Due to those reasons, there is a natural need to automatically recover the minified code with meaningful variable names. When the original code is not available, with such recovery, the minified JS code will be made accessible for code compre- hension as well as other maintenance activities such as code review, reuse, analysis, and enhancement. Recognizing that need, researchers have been introducing the automatically recovering tools for variable names in JS code. JSNice [17] is an automatic variable name recovery approach that represents the program properties and relations among program entities in a JS code as dependence graphs. It leverages advanced machine learning (ML) to recover missing variable names. Using also ML, JSNaughty [21] formulates the variable name recovery problem for JS code as a statistical machine translation from minified code to the recovered code. Despite of their successes, both approaches still suffer low accuracy and scalability issues with the use of computationally expensive ML algorithms.

In this work, we present JSNEAT, a data-driven, information retrieval (IR)-based approach to automatically recover variable name for minified JS code. The recovered names for variables must be natural in the context of the code and follow naming conventions. Thus, we conform JSNEAT in a datadriven direction, in which we aim to search for the name recovered for a minified variable in a large corpus of opensource JS code. We conjecture that meaningful, natural names of minified variables could be seen before in such corpus. Our key idea is to utilize the contexts for the variables in source code to search for its name. For the minified variables in a given a minified JS code, JSNEAT aims to match their contexts against the contexts in the corpus. If two contexts of two variables are similar, they should be named similarly, thus the variable's name in the corpus with the matched contexts should be a candidate name for the corresponding minified variable.

For a given minified variable $v$, we model three types of contexts. First, the name of a variable should be affected by its own properties and roles in the source code. For properties, if $v$ accesses to a method $m$ or a field $f$ in the code, then the recovered name for $v$ should be naturally compatible with the names of the method and the field. For example, the variable named dataTransfer is the receiver of the method call getData(). However, a variable that calls the method getData() cannot be randomly named in a regular program. For a role, if $v$ is used an argument of a method call $m$, the data type of $v$ must be compatible with $m$, thus, their names should also naturally be in conformance with one another. For example, in JQuery.trigger ( . . ), the first argument is either an event or an event type, thus, the name of the first argument should be consistent with a direct object of the verb trigger. 
Second, the name of a variable should be affected by the names of variables co-occurring in the same function. Several variables are used together to contribute to the current task of the function, thus, their names are naturally in concordance with one another. Observing some variable names, JSNEAT could predict the names for other co-occurring variables. It takes into account the naming of multiple variables at once considering such co-appearances. Finally, the third type of context is the current task of the function to which the variable belongs and contributes. The names of the variable should be relevant to the common task/purpose of the function. For example, the variables in a function getClipboardContent should serve the task of getting the content from the clipboard and have the names closely relevant to that task.

We combine the above contexts in the JSNEAT tool. We built a database to store the information about the variable names and the contexts extracted from a large corpus of opensource JS code. To recover the names for a given minified JS file, we use JSNEAT with three types of contexts to search for and rank the candidate names. We performed several experiments to evaluate JSNEAT on the dataset of $322 \mathrm{~K}$ JS files with almost $1 \mathrm{M}$ functions, and $3.5 \mathrm{M}$ variables with $176 \mathrm{~K}$ unique variable names. JSNEAT achieves high accuracy of $69.1 \%$, which is the relative improvements of $66.1 \%$ and $43 \%$ over two state-of-the-art approaches JSNice [17] and JSNaughty [21], respectively. A high percentage $(29.4 \%)$ of variables is recovered only by JSNEAT, while $4.7 \%$ and $3.6 \%$ of variables are recovered only by JSNaughty and JSNice, respectively. We reported that the time to recover for a file or for a variable with JSNEAT is twice as fast as with JSNice and $4 x$ as fast as with JNaughty, respectively. Importantly, JSNEAT's training time is $4 x$ faster than JSNice and $6 x$ faster than JSNaughty. This paper makes the following contributions:

1. JSNEAT: an IR-based, data-driven approach to recover variable names for minified JS code using 3 types of contexts;

2. An extensive comparative evaluation and analysis on JSNEAT's accuracy and running time to show that it outperforms the state-of-the-art approaches (See results in a website [10]);

3. A novel formulation of variable name recovery problem in minified JS code as an Information Retrieval problem.

\section{Motivation ANd Approach Overview}

Figures 1 and 2 show the original and minified versions of the JS function getClipboardContent in the vue-medium-editor project. The function is to retrieve the content of the clipboard. In the minified code, all local variables are randomly renamed with short and meaningless names, e.g., dataTransfer becomes $r$, data becomes $\mathrm{n}$, by a minification tool, e.g., UglifyJS [20]. This makes developers difficult to comprehend it.

Our goal is to assign meaningful names for the variables in the minified code. The name chosen for a variable in the code should be natural (unsurprising) in the context [21] and follow naming conventions [1], so that the de-minified code becomes easy to understand for developers.

To achieve this goal, we conjecture that the meaningful names of minified variables could be observed in a large cor-

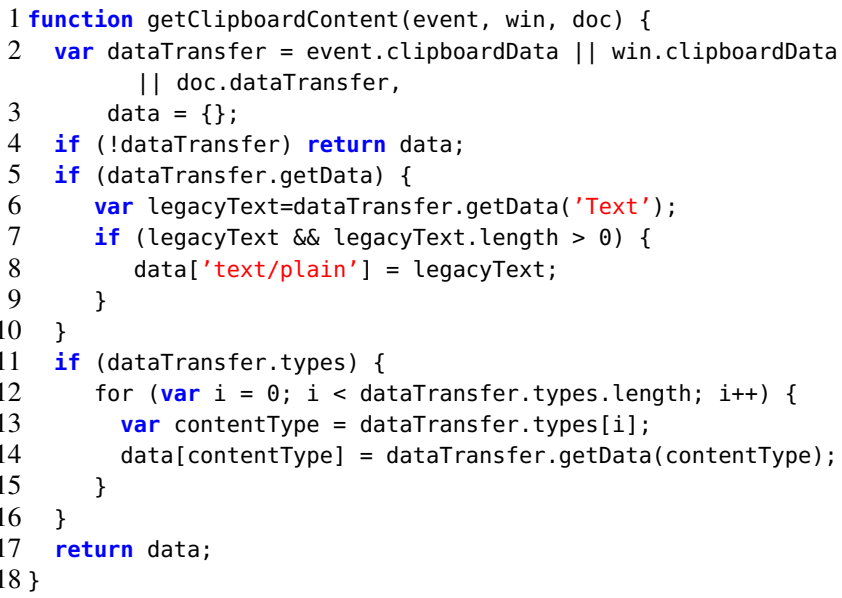

Figure 1: An Original Code from a Project in GitHub

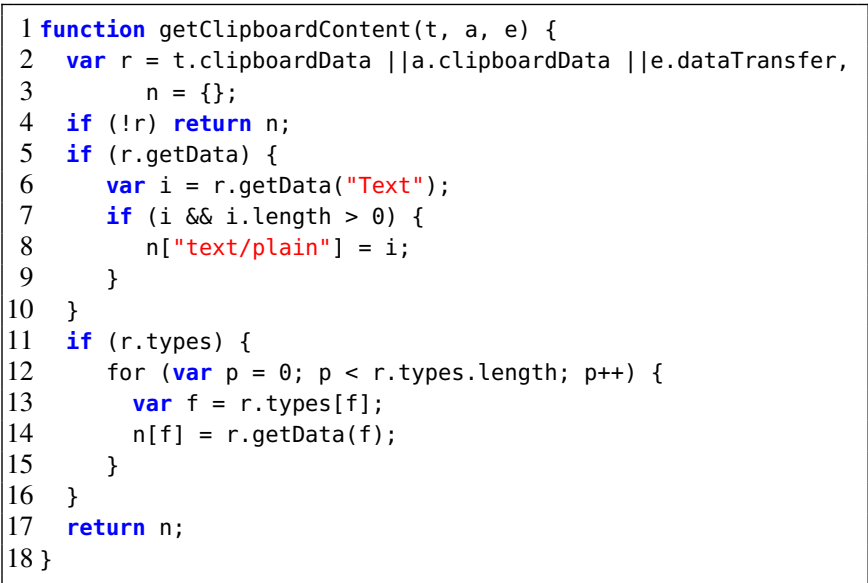

Figure 2: The Minified Code for the Code in Figure 1

pus of existing source code. This motivates us to conform our approach to a data-driven direction, where we learn the names from original source code to recover the names for variables in the minified code. Indeed, for the minified code in Figure 2, all original names are found in our experimental dataset that contains $322 \mathrm{~K}$ JS files collected from $12 \mathrm{~K}$ GitHub projects.

\section{A. Observations}

The name recovering process of variables in minified code is affected by multiple factors. Let us illustrate these factors through the following observations:

01. Each individual variable has certain properties and plays particular roles in the code. Thus, the name of a variable is intuitively affected by its properties and roles. The properties are the method calls or field accesses to which a variable of a certain type can access. If a method is called or a field is accessed by a variable, the name of the variable should be compatible with the method's or the field's name. For example, in our experimental dataset, the number of candidates that can 
call method getData () (lines 6 and 14) is only 7 out of 31 variables names found in a function named getClipboardContent. Such number is down to a single candidate if we additionally consider that it can also access the fields getData (line 5) and types (line 11). Thus, $r$ could be named as dataTransfer, which is the same name in the original code in Figure 1. For the variable $f$ that is created and assigned as an element of the array types [] at line 13, there are 4 candidates for such variable that can be used as an argument of the method named getData() (line 14). The number of candidates for $i$, which is the returned result of the call to getData () (line 6) and also has a field with the name length (line 7), is only 7 .

O2. In a function, a variable might collaborate with other variables to implement the function. Consequently, the recovering name for a variable might be influenced by the name of others. Intuitively, since the variables are used together, their names are often consistent with each other to achieve the common task in the function. In the example, in 28 possible pairs of candidates for $i$ ( 7 candidates) and $f(4$ candidates), there are only 2 pairs of candidates that are used to name two variables in the same function in our dataset. One of them is the correct pair, which is legacyText and contentType.

O3. Within a function, e.g., getClipboardContent, a variable name, e.g., contentType is affected by the specific task of the function that is described by the function's name [2]. This is intuitive because the names of variables are often relevant to the task that the variables are used in the code to achieve. Such task is typically described with a succinct function name. In Figure 1, the task of the function is to get the clipboard's content, thus, it is named getClipboardContent. In our dataset, there are 31 names being used to specify the variables in function getClipboardContent, e.g., data, dataTransfer, contentType. Meanwhile, the variable names students or salary have never been used in the function with that name.

Overall, these observations indicate that the names of the variables in a particular function not only depend on the task in which the variable is used to implement (called taskspecific context), but their names are also affected by their own properties and roles in the code (called single-variable usage context) and on the names of the other variables in the same function (called multiple-variable usage context).

\section{B. Approach Overview}

From the observations, we propose an IR-based, data-driven approach to recover the variables' names in a minified JS code based on the contextual information including singlevariable usage context (SVC), multiple-variable usage context (MVC), and task-specific context (TSC). We initially construct a database to store the variables' names and the corresponding context information extracted from a large corpus of JS code. To recover the names, given a minified JS code, we first use the $S V C$ and TSC information to find in our database the candidate names for each variable. Then, these candidates for each variable are ranked by the likelihood that they are used along with the candidates of other variables, in order to name the variables in the same function by using $M V C$.

\section{Single-Variable Usage Context (SVC)}

This section presents the single-variable context that we use in the name recovery process. The intuition for this context is that to recover the name of a variable, one could use its own context based on its own properties and roles in the code.

By properties of a variable, we refer to the methods or fields to which a variable of certain type can access. In the minified code, the names of the called methods and accessed fields are not minified. Thus, it could play the role of the pivots in recovering the variables' names. Importantly, due to nature of naming, the name of the variable should be compatible with the name of the method being called or the name of the field being accessed. Thus, they provide hints on the names of the variables. For example, in Figure 2, the only candidate that calls the method getData and accesses the field types is dataTransfer. Those names are compatible with each other. To learn compatible names, we follow a data-driven approach by learning from a large corpus of non-minified JS code.

By the role of a variable, we refer to its usage context with the method calls or field accesses that were not minified. For example, a variable could be an argument of a method call, or a variable could be assigned with the returned value from a method call or field access. The name of a variable used as an argument is often compatible with its type/role and, thus, coupled with the name of the method call itself. On line 14 of Figure 1, the argument contentType is in conformance with the method name getData. Thus, it helps recover the name of the minified variable $f$ on line 14 of Figure 2. Similarly, the name of a variable receiving the returned value of a method call or a field access should conform with the name of the method or field. Such conformance can be learned from a large corpus of non-minified code, and helps recover variable names.

\section{A. Property and Role Relations}

To realize the single-variable context for name recovery with properties and roles of a variable, we define two key relations: Property and Role. Those relations form the single-variable usage context for name recovery.

Definition 1. [Property Relation (PropRel)] Property relation represents the relationships between a variable and its fields or methods to which the variable can access or call.

A property relation between a variable $v$ and its property $\mathrm{p}$ is denoted by a triple $(v, p, t)$, where $\mathrm{t}$ is the type of relation, which can be either fieldAccess or methodCall. In Figure 2, a set of property relations for $r$ includes ( $r$, types, fieldAccess), ( $r$, getData, fieldAccess), $(r$, getData () , methodCall).

Definition 2. [Role Relation (RoleRel)] Role relation represents the relationships between a variable and the method calls or field accesses in its usages.

Since the names of methods or fields are not minified, we consider them as the pivots in the usage context for recovering names of the minified variables. We focus on the roles of a variable used as an argument in a method call or receiving 


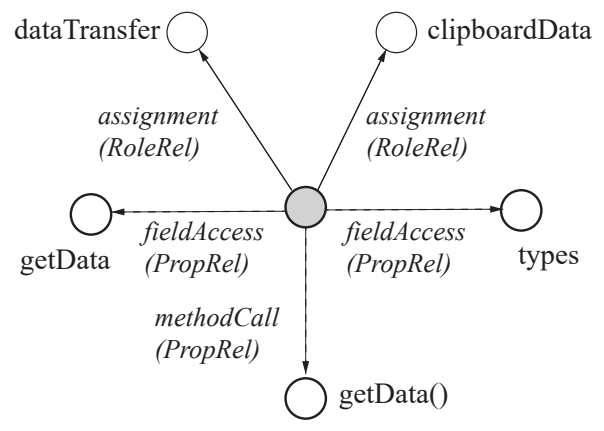

Figure 3: The Relation Graph of Variable $r$ in Figure 2

the value returned by a method call or field access. If we have $o . m(\ldots, v, \ldots), v=o . m(\ldots)$, or $v=o . f$, then there exist the role relations between $v$ and $m$, and between $v$ and $f$. The rationale is that the names of $m$ and its argument are often in conformance with each other, e.g., getData (contentType). Similar rationale is applied to the above assignments to $v$.

A role relation between a variable $v$ and a field/method $p$ is denoted by a triple $(v, p, t)$, where $\mathrm{t}$ is the type of role relation. A role relation could be either argument or assignment.

\section{B. Graph Representation of Single-Variable Context}

Definition 3. [Relation Graph] A relation graph $(R G)$ for a variable $v$ is a directed graph in the shape of a star to represent the single-variable usage context of $v$ with regard to its property and role relations with the fields and methods in its usage. The center vertex of the $R G$ represents the variable. The other vertices represent the methods/fields in method calls or field accesses, respectively, and are labeled with their names. Edges represent relations and are labeled with relation types.

Figure 3 shows the relation graph of the variable $r$, which includes a set of property relations: ( $r$, types, fieldAccess), ( $r$, getData, fieldAccess), ( $r$, getData(), methodCall), and a set of role relations: ( $r$, clipboardData, assignment), ( $r$, dataTransfer, assignment) in our example.

\section{Deriving Candidate Names Using Single-Variable Context}

Let us explain how we use the relation graphs to derive the ranked list of candidate names for a minified variable.

Our idea is that if two variables have the same/similar contexts, they are often named similarly. Given a minified function $f$, we first parse $f$ to produce a relation graph $G_{v}$ for each variable $v$. We then search for the single-variable contexts that are matched with the context of $v$ within a dataset $\mathcal{G}$ of the relation graphs built from a large corpus of open-source projects (We will explain how to build the dataset later).

Definition 4. [Single-Variable Context Matching] Two single-variable usage contexts for a minified variable $v$ and a variable $v^{\prime}$ in the dataset are considered to be matched if and only if their corresponding relation graphs are matched.

Definition 5. [Relation Graph Matching] A relation graph $G_{v}$ of a minified variable $v$ is considered as matched with relation graph $G_{v^{\prime}}$ of $v^{\prime}$ in $\mathcal{G}$ if and only if their graph matching score is equal or greater than a threshold $\varphi$.

Since RGs all have star shape, matching graphs can be done by matching their sets of edges.

Definition 6. [Relation Graph Matching Score] The graph matching score $\rho\left(G_{v}, G_{v^{\prime}}\right)$ between $G_{v}$ of a minified variable $v$ and a relation graph $G_{v^{\prime}}$ of $v^{\prime}$ is computed as the percentage of the number of edges in $G_{v}$ found in $G_{v^{\prime}}$.

A variable name might appear in multiple functions, so it might have multiple relation graphs. Thus, we define a name matching score considering all of those functions as follows.

Definition 7. [Single-Variable Score] Single-variable score represents how well name vn can be used for a minified name $v$ and is computed based on graph matching score:

$$
S C_{v, v n}=\max _{G_{v^{\prime}} \in \mathcal{G}_{v n}} \rho\left(G_{v}, G_{v^{\prime}}\right)
$$

where $\mathcal{G}_{v n}$ is the set of relation graphs of name vn that match $G_{v} \cdot \mathcal{G}_{v n}$ represents multiple usages of the name vn.

If a match is found, the name $v n$ of the variable $v^{\prime}$ in the matched relation graph in $\mathcal{G}$ is considered as a candidate name for $v$. There might exist many candidate names having similar contexts with the context of a variable $v$. The candidate names are ranked based on their name matching scores. The higher the name matching score, the higher the confidence of our model in using the name $v n$ of $v^{\prime}$ for $v$.

For example, when recovering the variable $r$ in Figure 2, by searching on the dataset, we found that the variables named dataTransfer and data0bj have the relation graphs matching with that of $r$. This implies that these variable names have been used in the past and they have similar SVC contexts with $r$. Therefore, $r$ could be recovered as dataTransfer or data0bj.

\section{Multiple-Variable Usage Context (MVC)}

Let us present how we define and use multiple-variable usage context. To achieve a specific task, developers use one or multiple variables in their code. Because the variables all play their roles in the code, their names are often relevant and consistent with one another in order to achieve the common task in the function to which they belong. For example, in Figure 1, the variables dataTransfer, contentType, and data serve their roles in the task to retrieve the content of a clipboard, and their names are naturally consistent with one another with regard to that task. In name recovery, we utilize such co-occurrences of variable names to recover the name for one variable while another one was recovered with the co-occurring name if the contexts of two variables allow.

\section{A. Multiple-Variable Score}

To formulate the co-occurrence of variable names, we define the association score for a set of variable names, which represents how likely those names appear together in a function. Assume that we have a set of $n$ variable names, and the name 
of $i^{t h}$ variable is $v n_{i}$. The association score for a set $S$ of $n$ names $\left(v n_{1}, v n_{2}, \ldots, v n_{n}\right)$ is computed as follows.

$$
\operatorname{assoc}(S)=\frac{N_{v n_{1} \cap v n_{2} \cap \ldots \cap v n_{n}}}{N_{v n_{1} \cup v n_{2} \cup \ldots \cup v n_{n}}}
$$

where $N_{v n_{1} \cap v n n_{2} \cap \ldots \cap v n_{n}}$ is the number of functions that contain all the names $\left(v n_{1}, v n_{2}, \ldots, v n_{n}\right)$ in the corpus. $N_{v n_{1} \cup v n_{2} \cup \ldots \cup v n_{n}}$ is the number of functions that contain at least one of the names $\left(v n_{1}, v n_{2}, \ldots, v n_{n}\right)$ in the corpus.

For a set of $n$ recovered names, we define a Multiplevariable score $(M C)$ that represents the likelihood of those variable names to be assigned to the variables based on MVC. $M C$ can be computed using the association score. However, due to the fact that not all possible sets of $n$ names appear together in a corpus, we compute $M C$ based on the associations of all subsets of the size $J$ with $J \leq n$, as follows:

$$
M C_{v n_{1}, v n_{2}, \ldots, v n_{n}}=\frac{\sum_{i=1}^{n_{\text {subJ }}} \operatorname{assoc}\left(S_{i}\right)}{n_{\text {subJ }}}
$$

where $S_{i}$ is a subset of size $J$ of $\left(v n_{1}, v n_{2}, \ldots, v n_{n}\right) ; n_{s u b J}$ is the number of such subset; and $\operatorname{assoc}\left(S_{i}\right)$ is the association score of all variable names in set $S_{i}$ (computed by Formula 2).

\section{B. Deriving Candidate Names Using Multi-Variable Context}

This section presents our algorithm to derive candidate names using multiple-variable usage context. The algorithm takes as input a set of minified variables in a JS code in which each variable has a set of candidate names (derived using the single-variable context as explained in Section III-C or using task-specific context in Section V). The output is the ranked list of the results with associated scores. Each result is a set of the recovered names for all of the given variables.

1) Design Strategies: In developing our algorithm, we face three key challenges. First, each variable might have a large number of candidates, thus, there are an exponential combination among variables' names. How would we deal with such complexity to make our algorithm scale? Second, given a set of minified variables needed to be recovered, which one should JSNEAT start? This is important since if the algorithm does not recover well the first variable, this would affect much to the accuracy of recovering the next variables. Finally, in which recovery order for the variables in a function would it be beneficial from multiple-variable usage context? To address those questions, we have the following design strategies.

S1. Pruning with Beam Search. To deal with the scalability issue of the exponential combination among all possible names of variables, we use the Beam Search strategy: at a step during name recovery, our algorithm keeps only the best $K$ sets of partially recovered results according to the association scores. This would help to reduce significantly the number of partially recovered sets that need to be considered.

S2. Starting with Variable with Most Contextual Information. A naive answer is to use the appearance order of the variables in the code. However, the first variable in the code might not be the one that we have sufficient information to recover its name. Thus, we follow the idea of using context to

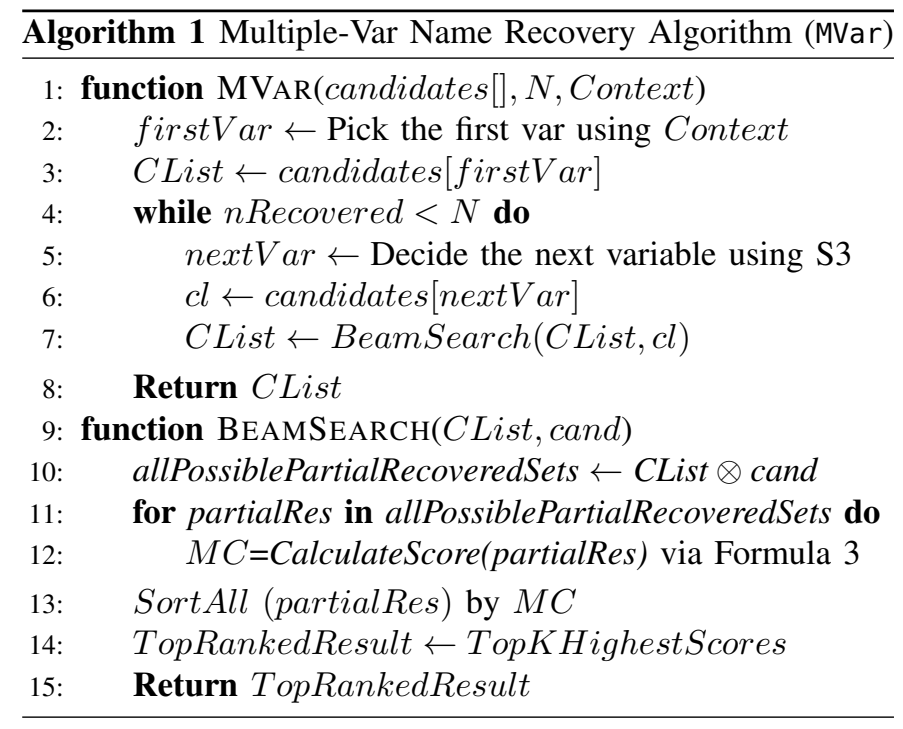

decide the initial variable for name recovery. Our intuition is that the more context information a variable has, the more chance we have in correctly recovering its name. JSNEAT starts with the variable having the most single-variable usage information. That is, the variable has the most relations with method calls and field accesses. The appearance order is used to break the tie if multiple variables have the same number of relations in their single-variable contexts.

S3. Selecting Next Variable with Greedy Strategy. After one or multiple variable names are recovered, we need to determine which variable to recover next. The appearing order in the code might not give us the optimal one. The variable with the most single-variable context might not work either since it might not go together well with others. In JSNEAT, we select the next variable $v$ with the list of candidate names vns that gives us the best partially recovered result. That is, together with the selected names for the previously recovered variables, the best possible choice for $v$ would give the highest score with respect to the likelihood of the co-occurrences of the recovered variables including $v$. This is a greedy strategy that favors the variable and its candidate name that has most co-appearances with the previously recovered variable names. It helps avoid considering all possible candidate names for all the variables. We use the phrase "partially recovered result" because only a subset of all variables including $v$ is recovered for their names, while other variables have not been processed.

2) Detailed Algorithm: Algorithm 1 shows the pseudo-code for our algorithm, MVar, to derive names using the multiplevariable contexts. Given a set of $N$ minified variables in which each variable has a set of candidate names candidates (provided by a Context, e.g., Single-variable or Task-specific contexts), MVar determines the first variable to start. Using the strategy S2, it chooses the first minified variable with the highest score according to the context (line 2). For example, if the single-variable context is chosen, Formula 1 is used. All the candidates for the first variable are initially stored in the current candidate list CList. Then, the algorithm 
iterates to recover the variable names until all the variables are recovered (lines 4-7). At a step of the iteration, assume that it has recovered $n$ Recovered variables. Using the strategy $\mathrm{S} 3$, the next variable nextVar is chosen such that together with the selected names for $n$ Recovered previously recovered variables, the possible names for nextVar will give the highest $M C$ score (Formula 3) considering the co-occurrences of currently recovered variables (lines 5-6).

After selecting the next variable, MVar performs beam search by first generating all possible names for the (nRecovered + 1) variables with combining the name candidates for nextVar and CList (line 10). Each of those sets of names represents a partially recovered result for those (nRecovered +1$)$ variables. The scores of all of those sets are computed (lines 11-12) using Formula 3. Then, we keep only the best $K$ sets of results with highest scores and then store them in CList (lines 13-14). MVar stops when all variables have been recovered. CList is returned as the list of best $K$ sets of variable names for all the variables in the input (line 8).

In our example, using the single-variable context and/or the task-specific context, we have the set of name candidates for each variable, e.g., $r$ : (dataTransfer, dataContent,...), $f:($ elementType, dataType, contentType, ... $), p:(i, j, \ldots)$, n:(data, cacheData, dataContent,...), etc. The variable $r$ is chosen first since its single-variable context has most relations. All the name candidates for $r$ are stored in CList. After that, the next variable is $\mathrm{n}$ because in all the candidate names of the non-yet-recovered variables, the name data for $\mathrm{n}$ when appearing with the current candidate name of $r$ gives the highest score. In this case, the candidate name of $r$ is dataTransfer. Then, all the sets of variable names for $(r, n)$ include (dataTransfer, dataContent), (dataTransfer, cacheData), (dataTransfer, data), etc. Ranking these partially recovered results with Formula 3 and keeping only the top $K$ ones, CList includes (dataTransfer, data), (dataTransfer, dataContent),... The next variable for recovery is $f$. The process continues until all variables are recovered. CList results are returned as the output.

\section{TASK-SPECIFIC CONTEXT (TSC)}

In a program, a function has its functionality and is written to realize a specific task. Each variable used in that function plays a certain role toward that task. Thus, the names of variables are relevant to the task of the function and often consistent with one another. The task of a function is typically described by a succinct name of the function. To derive a variable name using the task context, we use the association relation to compute how likely a variable name appears within a function with a particular name. Given a variable name $v n$ and a function name $f n$, Task Context score (TC) represents the likelihood that the name $v n$ appears within the body of a function named $f n$. We utilize fuzzy set theory [12] as follows.

$$
T C_{v n, f n}=\frac{N_{v n, f n}}{N_{v n}+N_{f n}-N_{v n, f n}}
$$

where $N_{v n, f n}$ is the number of functions in the corpus in which $v n$ and $f n$ are observed together; $N_{v n}$ is the number of functions in which $v n$ is used; and $N_{f n}$ is the number of functions named $f n$. As seen in Formula 4, the value of TC is between $[0,1]$. The higher the value $T C_{v n, f n}$, the higher the likelihood that the variable name $v n$ appears in the function $f n$. In Figure 1, using our experimental dataset, we can compute $T C$ score between the function name getClipboardContent and the variable name dataTransfer as $T C=\frac{3}{21+5-3}=0.13$.

A function name might contain multiple tokens, e.g., get, Clipboard, Content in getClipboardContent. Each token contributes to an aspect to emphasize the common task of the function. A variable might be relevant to one specific aspect of the task. Thus, if we tokenize the function names, we can account for those cases. By tokenizing, a function name $\mathrm{fn}$ could be represented by a set of key tokens $S=\left\{t_{1}, t_{2}, t_{3} \ldots\right\}$ (stopwords are removed) and the $T C$ score between a variable name $v n$ and a function name $f n$ is computed as follows:

$$
T C_{v n, f n}=\max _{t \in S} \frac{N_{v n, t}}{N_{v n}+N_{t}-N_{v n, t}}
$$

where $S$ is the set of key tokens of $f n ; t$ is a token in $S$; $N_{v n}$ is the number of functions in which $v n$ is used; $N_{t}$ is the number of functions containing token $t$; and $N_{v n, t}$ is the number of functions in which $v n$ and $t$ occur together.

\section{VARIABle Name Recovery with CONTEXts}

This section presents JSNEAT, our approach to recover the variable names in minified code using the combination of those above contexts. Given a minified JS file, whose variables have been minified, JSNEAT produces a recovered JS file in which all variables are recovered with meaning names.

Algorithm 2 shows the pseudo-code for JSNEAT. First, JSNEAT builds the relation graph representing single-variable context (SVC) for each variable in a function and derives the candidate list. It then computes the task-specific context (TSC) for each variable and derives the corresponding candidate list. The two candidate lists produced by the contexts are combined into a new list in which the likelihood that a candidate name $v n$ is assigned to a variable $v$ is computed as follows.

$$
S T_{v, v n}=\alpha \times S C_{v, v n}+\beta \times T C_{v n, f n}
$$

where $f n$ is the function name; $T C_{v n, f n}$ is task context score between $v n$ and $f n ; S C_{v, v n}$ is the name matching score between $v n$ and $v$, and $\alpha$ and $\beta$ are weighting parameters, representing the importance of the contexts.

After this step, for each variable, we have a candidate list (ST List) in which each candidate name has a score. JSNEAT then uses STList as the input for MVar (Algorithm 1) to compute the top-ranked sets of names for all variables.

Note that the scoring function for a partially recovered result in MVar algorithm (Formula 3) needs to be adjusted to account for the above combined score $S T$ as follows.

$$
\begin{aligned}
& M C_{v n_{1}, v n_{2}, \ldots, v n_{n}}=\gamma \times \frac{\sum_{j=1}^{n_{\text {subJ } J}} \operatorname{assoc}\left(S_{j}\right)}{n_{\text {subJ }}} \\
& +\theta \times \frac{S T_{v_{1}, v n_{1}}+S T_{v_{2}, v n_{2}}+\ldots+S T_{v_{n}, v n_{n}}}{n}
\end{aligned}
$$

where $\gamma$ and $\theta$ are weighting parameters. 


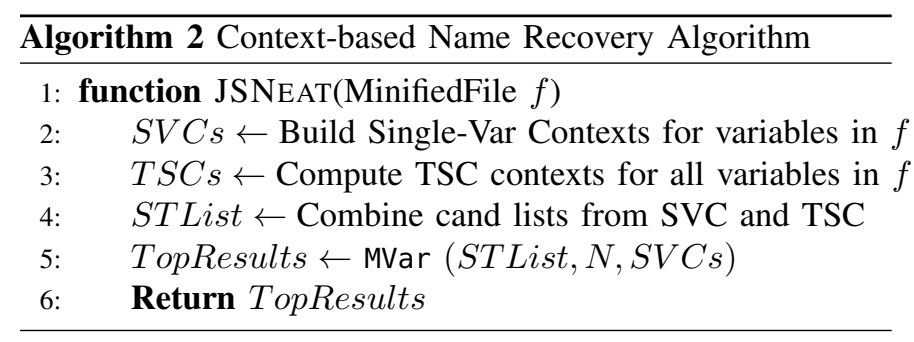

Table I: Data Collection

\begin{tabular}{c|c|c|c}
\hline Category & Test Corpus & Training Corpus & Total \\
\hline Files & $2 \mathrm{~K}$ & $320 \mathrm{~K}$ & $322 \mathrm{~K}$ \\
Functions & $6 \mathrm{~K}$ & $961 \mathrm{~K}$ & $967 \mathrm{~K}$ \\
Variables & $19 \mathrm{~K}$ & $3481 \mathrm{~K}$ & $3.5 \mathrm{M}$ \\
Unique variable names & $5 \mathrm{~K}$ & $171 \mathrm{~K}$ & $176 \mathrm{~K}$ \\
Variables per file & 9.5 & 10.94 & 10.93 \\
\hline
\end{tabular}

\section{EMPirical Methodology}

To evaluate JSNEAT, we answer the following questions: RQ1: Comparative Study. How accurate is JSNEAT in name recovery for minified JS code and how is it compared with the state-of-the-art approaches, JSNice [17] and JSNaughty [21]? RQ2: Context Analysis. How do different combinations of contexts contribute to JSNEAT's accuracy in different settings? RQ3: Sensitivity Analysis. How do various factors affect the accuracy, e.g., data's sizes, thresholds, parameters, etc.? RQ4: Time Complexity. What is JSNEAT's running time?

\section{A. Corpora}

We collected a corpus of 12,000 open-source JS projects from GitHub with highest ratings. For comparison, we followed the same procedure in previous work [17], [21] to collect and clean up data. We removed all duplicate files to avoid overlapping when testing. We also removed the alreadyminified files because they will not help in training. Table I shows our dataset's statistics. As seen, the number of unique variable names is much smaller than that of variables. Thus, such repetition in names would help our data-driven approach.

In our comparative study, we used the same experimental setting as in JSNice [17] and JSNaughty [21] by randomly splitting the dataset into training and test corpora. In particular, to build Testing Corpus, we randomly sampled 2K JS files in the dataset. The remaining $320 \mathrm{~K}$ files were used as Training Corpus. The level of sizes of testing and training data (Table I) is comparable with that of the experimental studies in existing tools [17], [21]. We minified the files using the minifying tool UglifyJS [20], and used the original files as oracle.

To build the relation graphs, we used Rhino to parse the JS files and extract the context information. Table II shows the statistics of our dataset $\mathcal{G}$ of relation graphs.

\section{B. Evaluation Setup}

1) Comparative Study: For a tool under study, we trained it with the Training Corpus and tested it against the Testing Corpus. For JSNice, we used the publicly available tools on their website [11] with default parameters. For JSNaughty, we
Table II: Database of Relation Graphs

\begin{tabular}{c|c}
\hline Category & Quantity \\
\hline Total number of graphs & $3.5 \mathrm{M}$ \\
Mean number of graphs per file & 10.93 \\
Mean number of graphs per function & 3.62 \\
Min/Mean/Max number of edges per graph & $1,2.2,41$ \\
\hline
\end{tabular}

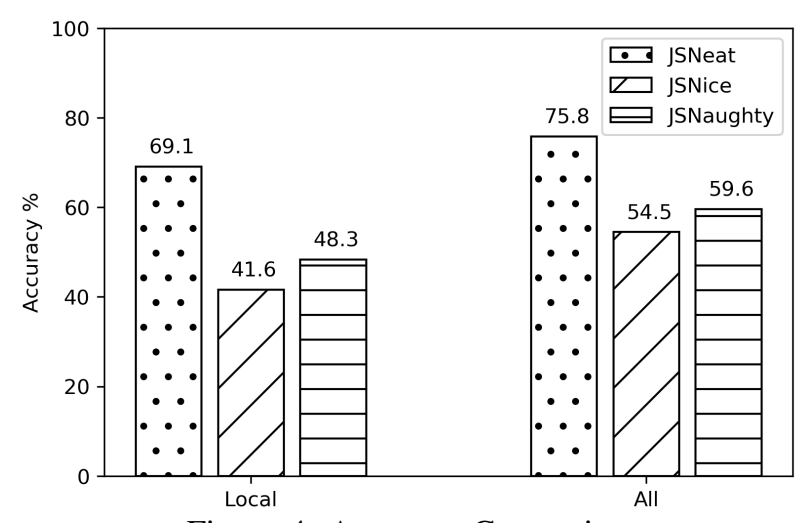

Figure 4: Accuracy Comparison

trained the translation and language models, and Nice2Predict framework following the instructions. We did not run their tools in 10-fold cross validation due to a long running time.

2) Context Analysis: We study the impact of different contexts, we created different variants of JSNEAT with various combinations of contexts and measured their accuracies. We used the 10-fold cross-validation setting on the entire corpus: $90 \%$ of the files ( 9 folds) are used for training and $10 \%$ of the files (one fold) for testing, and we repeated testing for each of the 10 folds and training with the remaining folds. We also performed 10-fold cross validation on the project basis.

3) Sensitivity Analysis: To study different factors that have impact on JSNEAT's accuracy, in our entire dataset, we randomly chose one fold for testing and the remaining 9 folds for training. We studied the following factors: relation graph size, type of relation edges, thresholds, beam sizes, different degrees of associations, different weight parameters, and data size.

\section{Procedure and Metrics}

To measure the accuracy of a tool, we used UglifyJS [20] to minify the given JS files, and used the minified code as the input for the tool under study. We then compared the resulting names from the tool against the original names. Specifically, the tool is considered to correctly recover the name of a variable $v$ if the recovered name $v n$ is matched exactly with its original name. For $v$, if matching, we count it as a hit, otherwise, it is a miss. Accuracy is measured by the ratio between the total number of hits over the total number of cases.

\section{EMPIRICAL RESULTS}

\section{A. Accuracy Comparison (RQ1)}

In this study, we evaluate JSNEAT's accuracy and compare it with JSNice [17] and JSNaughty [21]. As seen in Figure 4 , for local variables, JSNEAT achieves high accuracy of 


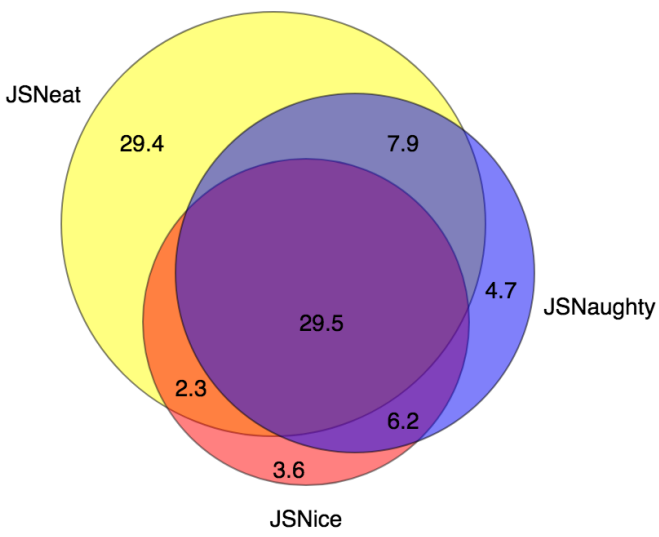

Figure 5: Overlapping among Results from the Tools

69.1\%: relative improvements of $66.1 \%$ and $43 \%$ over JSNice and JSNaughty, respectively. The absolute improvements are $27.5 \%$ and $20.8 \%$, respectively. For all variables (local and global ones), JSNEAT achieves even higher accuracy: $75.8 \%$, the relative improvements of $39 \%$ and $27 \%$ over JSNice and JSNaughty, respectively. The absolute improvements are $21.3 \%$ and $16.2 \%$, respectively. Note that global variables are not minified, we computed the accuracy for all variables for the completeness purpose. From now on, using the terms "variables", we refer to the recovery accuracy for local variables.

We further analyze the overlapping between the results from three tools. The Vein diagram in Figure 5 shows the percentages of variable names that are correctly recovered. As seen, a high percentage $\mathbf{2 9 . 4 \%}$ ) of variables is recovered only by JSNEAT, while $4.7 \%$ and $3.6 \%$ of variables are recovered only by JSNaughty and JSNice, respectively. Meanwhile, there are $14.5 \%$ of the variables that are correctly recovered only by JSNice or JSNaughty, and not by JSNEAT.

We also analyzed which contexts in JSNEAT contribute to those $29.4 \%$ correctly recovered names. To do that, we deactivated each of the three contexts. When MVC is disabled, the percentage of variables that are correctly recovered only by JSNEAT decreases by $7.4 \%$ compared to the full version. However, when TSC is disabled, the accuracy drops dramatically from $29.4 \%$ to $7.6 \%$. Taking a deeper look in the cases of $29.4 \%$, we found out that $19 \%$ of variables that were recovered correctly when using all contexts become incorrectly recovered ones when TSC is off. $83 \%$ out of that $19 \%$ come from the function with a single variable. MVC certainly would not help in those cases because it needs the contexts from other variables. In fact, in such functions, the function names are quite relevant to the variable names. While SVC does not have enough information to rank the correct name on the top, TSC provides useful information to help in those cases. For example, function responseJson in project $\mathrm{mf}$-geoadmin3 uses a variable named response, that was minified into $x$. Using only SVC and MVC, the correct name response is ranked at position 5, but adding TSC, response is ranked at the top. In the next experiment, we deactivated the single-
Table III: Impact of Contexts on Accuracy and Recovery Time

\begin{tabular}{||c|c|r|r||}
\hline & Combination of Contexts & Acc (\%) & Time (ms) \\
\hline 1 & Task (TSC) & 5.2 & 1.3 \\
2 & SingleVar (SVC) & 33.7 & 1.5 \\
3 & Task + SingleVar & 47.3 & 2.1 \\
4 & Task + MultiVar (MVC) & 9.3 & 2.3 \\
5 & SingleVar + MultiVar & 37.8 & 2.6 \\
6 & Task + SingleVar + MultiVar (= JSNEAT) & 63.1 & 3.2 \\
\hline
\end{tabular}

variable context $(S V C)$, and the percentage of variables that are correctly recovered only by JSNEAT decreases to $0.2 \%$. This means that SVC with property and role relations with the pivots in the code contributes most to that $29.4 \%$ of the cases that were not recovered correctly by the other tools.

\section{B. Context Analysis Evaluation Results (RQ2)}

As seen in Table III, using only task-specific context (TSC) (line 1), accuracy is low because all the variables in the same function have the same chance to be recovered with a certain name. In contrast, the single-variable context (SVC) achieves much higher accuracy (33.7\%). This is reasonable since SVC provides more detailed context for individual variables such as the relations to surrounding method calls and field accesses.

Combining TSC and SVC provides an additional improvement of $13.4 \%$ over the tool with only SVC (lines 2 and 3). We found that several correct candidate names that were ranked in the 2nd-4th positions become the top candidates with the addition of TSC. In contrast, the combination of TSC and MVC yields only slight improvement over TSC (5.2 to 9.3). The reason is that MVC takes the lists of candidate names as its input and such lists were not initially of high quality (only $5.2 \%$ accuracy), leading to low accuracy. For the combination of SVC and MVC, the improvement is $4.1 \%$ over SVC (lines 2 and 5). We found that those $4.1 \%$ of cases, the co-occurrences of variable names help rank them in the top positions. Comparing lines 3 and 5, adding TSC to SVC improves almost $10 \%$ more than adding MVC to SVC. Further analyzing, we found that such improvement from TSC is for the cases in which 1) the given JS function has only one variable (MVC cannot help) and/or 2) the SVC has only one relation (SVC did not perform well with little surrounding context).

Finally, combining three contexts, JSNEAT achieves the highest accuracy. Compared to TSC+SVC (lines 3 and 6), JSNEAT relatively improves $\mathbf{3 3 . 4 \%}$ (15.8\% absolute improvement). This is reasonable since the two contexts TSC and SVC alone achieve the highest accuracy among all the combinations of two contexts. Thus, they give MVC algorithm the initial candidate lists for variables with higher quality. Then, MVC with the co-occurrence information among variables helps an additional improvement of $15.8 \%$. Moreover, comparing lines 5 and 6, TSC helps improve $25.3 \%$ since TSC helps in the cases of single-variable functions or single-edge RGs.

To evaluate JSNEAT's consistency in achieving high accuracy, we performed 10-fold cross validation. As seen in Table IV, the accuracies for all the folds are stable (61.9-63.8\%), with the recovery time of $2.92 \mathrm{~ms}$ for a file. The results for 10fold cross validation on project basis are similar (not shown). 
Table IV: 10-fold Cross-Validation Evaluation on JSNEAT

\begin{tabular}{|c||c|c|c|c|c|c|c|c|c|c||c||}
\hline Test Fold & 0 & 1 & 2 & 3 & 4 & 5 & 6 & 7 & 8 & 9 & Mean \\
Acc $(\%)$ & 63.1 & 63.0 & 63.1 & 61.9 & 63.3 & 62.7 & 63.8 & 63.4 & 62.9 & 62.5 & 62.9 \\
Time & 2.9 & 2.92 & 2.86 & 2.83 & 2.95 & 2.99 & 2.92 & 2.95 & 3.01 & 2.85 & 2.92 \\
\hline
\end{tabular}

Table V: Impact of Relation Graphs' Sizes on Accuracy

\begin{tabular}{||c||c|c|c|c|c|c|c||}
\hline Number of edges & 1 & 2 & 3 & 4 & 5 & $>5$ & All \\
\hline \% of graphs & 46.4 & 24.8 & 12.8 & 6.6 & 3.5 & 5.7 & 100 \\
\hline Accuracy (\%) & 58.5 & 65.7 & 67.4 & 68.4 & 68.7 & 69.8 & 63.1 \\
\hline
\end{tabular}

\section{Sensitivity Analysis Evaluation Results (RQ3)}

1) Impact of Relation Graphs' Sizes: To measure the impact of the sizes of SVC, we selected in the corpus only certain sizes of RGs (measured by the number of edges). As seen in Table V, the more relations in SVC to be considered (more edges a relation graph has), the more accurate a variable name can be recovered. When having more than 4 edges, accuracy becomes stable at a high level and gradually increases. This also reaffirms our strategy $\mathrm{S} 1$ in selecting the first variable with most connecting edges in RGs.

2) Impact of Type of RG Edges: By omitting only certain type of edges in RGs for SVC, we measured the impact of each type of relations on accuracy. In Table VI, the lower the accuracy, the higher the impact the corresponding relation has. As seen, all the relation types contribute nearly equally to JSNEAT. If one type of relation is not considered, accuracy drops from $63.1 \%$ to around $45 \%$. The argument relation has a slightly higher contribution than the others.

3) Impact of Threshold for Graph Matching: We evaluated the impact of the threshold $\varphi$ used to measure the similarity between two RGs (i.e., two SVCs). To do that, we used only SVC to recover variable names with varied $\varphi[0.5-1.0]$. As seen in Table VII, when $\varphi=0.8$, the accuracy is at the highest. With $\varphi<0.8$, the number of variables whose contexts are matched with the minified variable is large, and the correct name was not ranked at the top. When $\varphi>0.8$, the condition is too strict and the correct names were dropped because it is not easy to find a completely matched context.

4) Impact of Beam Size: In JSNEAT, beam size is used to deal with the large combinations of possible names. Figure 6 shows the accuracy and running time per variable when we varied the beam sizes. As seen, when the beam size is small, the accuracy is very low. It is expected because pruning occurs frequently, the number of results that were kept is smaller, and the best candidates might be dropped out of the beam stack. As the beam size is increased, accuracy increases and reaches the highest point (around 63\%) with the beam size of 30. Accuracy becomes stable when the beam size is greater than 30 . The reason is that almost all the correct names are observed in the top 30 results. Therefore, when we increase beam size over 30 , accuracy is not affected anymore. Regarding the running time, the higher the beam size, the larger the number of candidate results, and the higher the running time. Thus, we used 30 for the beam size in other experiments.

5) Impact of Association Scores: In MVC, JSNEAT considers the co-occurrences of $J=2,3$, etc or $n$ variable names. The
Table VI: Impact of Relation Types in RGs on Accuracy

\begin{tabular}{||c||c|c|c|c||}
\hline & argument & assignment & fieldAccess & methodCall \\
\hline Accuracy (\%) & $42.9 \%$ & $44.5 \%$ & $48.5 \%$ & $45.7 \%$ \\
\hline
\end{tabular}

Table VII: Impact of Threshold $\varphi$ on Accuracy

\begin{tabular}{||c||c|c|c|c|c|c||}
\hline Threshold $\varphi$ & 0.5 & 0.6 & 0.7 & 0.8 & 0.9 & 1.0 \\
\hline Accuracy $(\%)$ & 27.1 & 27.4 & 30.6 & $\mathbf{3 3 . 7}$ & 31.2 & 30.8 \\
\hline Time & 1.8 & 1.7 & 1.5 & 1.5 & 1.4 & 1.4 \\
\hline
\end{tabular}

value of $J$ shows how many associations of variable names that we need to have high accuracy. In Table VIII, using highdegree association, accuracy decreases gradually since finding co-occurrences of $n$ variable names has a lower probability than finding the co-occurrences of $n-1$ variable names, i.e., the co-occurrence condition is too strict. The decrease in accuracy is not much since the high-degree association affects only a smaller set of cases with higher numbers of variables in a function. As expected, running time increases.

6) Impact of Parameters in Context Combinations: To combine multiple contexts, we use parameters to put weights on each of them, e.g., $\alpha$ for SVC, $\beta$ for TSC, and $\gamma$ for MVC. We varied their values to observe the impacts of the contexts. In Table IX, when combining SVC and TSC, if the weight of SVC is higher, accuracy is higher. In a function, task context plays an equal role to all variables in that function, while SVC provides directly related information to the variable. Combining SVC, TSC, and MVC, the higher $\gamma$, the higher the accuracy. This means that MVC contributes more important information than SVC and TSC in the Formula 7.

7) Impact of Training Data Size: To measure impact of data size, we used one fold for testing and increased the sizes of the Training dataset by adding one fold at a time until 9 remaining folds are added for training. We ran JSNEAT on each training dataset with the best settings for SVC and MVC, and two settings of TSC: tokenizing or using full function names. In Table $\mathrm{X}$, the accuracies in both settings increase linearly and consistently with the training size. With a small size, tokenizing function names gives better accuracy than using full names. However, when the number of training folds is more than 5 folds, using full function name is better. The reason is that when the data's size is large enough, the probability of coappearances between a function name and a variable name is higher, the candidate names for a variable can be found better. When data's size is small, JSNEAT might not see a variable name and a function name appearing together, then tokenizing function names would give more useful context.

\section{Time Complexity (RQ4)}

All experiments were run on a Linux server with 20 Intel Xeon $2.2 \mathrm{GHz}$ processors, 256GB RAM. In Table XI, the time to recover for a file or for a variable with JSNEAT is twice as fast as with JSNice and $4 x$ as fast as with JNaughty. More importantly, JSNEAT's training time is $4 x$ faster than JSNice and $6 x$ faster than JSNaughty. This can be achieved due to the nature of information retrieval in JSNEAT, in comparison to machine learning in JSNice and JSNaughty. 


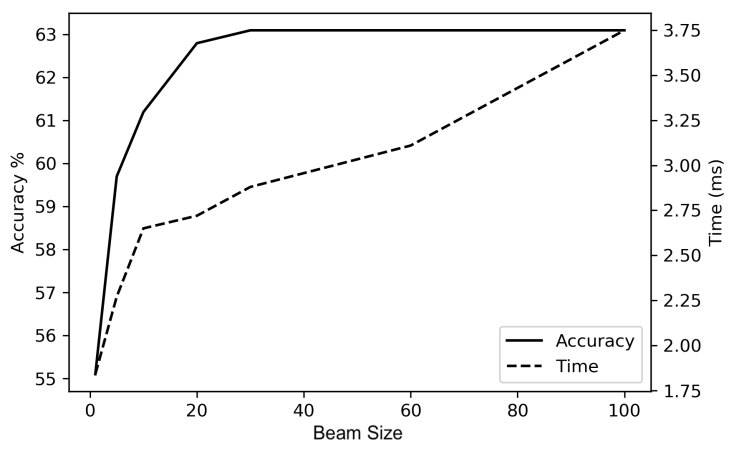

Figure 6: Impact of Beam Size on Accuracy and Running Time

Table VIII: Impact of Assoc Score $J$ on Accuracy and Time

\begin{tabular}{||c||c|c|c||}
\hline$J$ & Accuracy $(\%)$ & Time $(\mathrm{ms})$ & Perc. Found $(\%)$ \\
\hline Pairwise $(J=2)$ & 63.1 & 2.88 & 68.5 \\
\hline Triple $(J=3)$ & 61.8 & 3.11 & 55.2 \\
\hline$J=4$ & 60.2 & 3.26 & 25.7 \\
\hline$J=6$ & 58.6 & 3.41 & 14.9 \\
\hline$J=8$ & 57.5 & 3.45 & 5.8 \\
\hline$J=10$ & 57.4 & 3.47 & 2.7 \\
\hline
\end{tabular}

\section{E. Limitations and Threats to Validity}

1) Limitations: First, as a data-driven approach, unseen data affects our accuracy. For example, with 1-fold training data, $33.4 \%$ of minified names have not been observed. Second, for the task context, JSNEAT did not work well for functions with general names, e.g., next, find, etc. More sophisticated solution could involve topic modeling [4] on the function body. Third, for MVC, greedy strategy might not achieve the optimal result. Finally, if two variables in the same function are assigned with the same name (e.g., same SVC, MVC, and TSC), we randomly pick different names. Program analysis could be applied here to improve accuracy.

2) Threats to Validity: Our corpus of JS code might not be representative, however, we chose a large corpus with the size comparable with those in previous studies. We used only the tool Uglify to minify the code, which was also used in JSNice and JSNaughty. We do not study the usefulness involving human subjects. However, for comparison, we used the same experimental settings as in JSNice [17] and JSNaughty [21].

\section{RELATED WORK}

JSNEAT is closely related JSNice [17] and JSNaughty [21]. JSNice [17] uses the graph representation of variables and surrounding program entities via program dependencies. It infers the variable names as a problem of structured prediction with conditional random fields (CRFs) [17]. In comparison, first, while JSNice uses ML, JSNEAT is IR-based in which it searches for a list candidate names in a large code corpus. Second, JSNEAT considers not only the impacts of surrounding program entities in SVC, but also task and multiple-variable contexts. Third, with CRF, JSNice is effective when variables have more dependencies, and less effective with the functions
Table IX: Sensitivity Analysis on Combination Parameters

\begin{tabular}{||c|c|c||c|c|c||}
\hline$\alpha$ & $\beta$ & Accuracy (\%) & $\theta$ & $\gamma$ & Accuracy (\%) \\
\hline 1 & 0 & 45.4 & 1 & 0 & 57.9 \\
0.25 & 0.75 & 45.9 & 0.75 & 0.25 & 58.5 \\
0.5 & 0.5 & 46.5 & 0.5 & 0.5 & 60.2 \\
0.75 & 0.25 & 47.3 & 0.25 & 0.75 & 61.7 \\
0 & 1 & 46.7 & 0 & 1 & 63.1 \\
\hline
\end{tabular}

Table X: Impact of Training Data's Size on Accuracy

\begin{tabular}{||c||c|c|c|c|c|c|c|c|c||}
\hline \#Folds & 1 & 2 & 3 & 4 & 5 & 6 & 7 & 8 & 9 \\
\hline Acc.Full & 40.1 & 47.3 & 52.5 & 54.9 & 56.4 & 58.2 & 60.0 & 62.2 & 63.1 \\
\hline Acc.Token & 42.3 & 48.5 & 53.6 & 55.1 & 56.5 & 57.3 & 57.9 & 58.5 & 59.1 \\
\hline
\end{tabular}

Table XI: Running Time Comparison

\begin{tabular}{||c||c|c|c||}
\hline Metric & JSNEAT & JSNice & JSNaughty \\
\hline Training & $2 \mathrm{~h} 05 \mathrm{~m}$ & $8 \mathrm{~h} 35 \mathrm{~m}$ & $12 \mathrm{~h} 25 \mathrm{~m}$ \\
Per-file Recovery & $32 \mathrm{~ms}$ & $72 \mathrm{~ms}$ & $129 \mathrm{~ms}$ \\
Per-variable Recovery & $2.9 \mathrm{~ms}$ & $6.6 \mathrm{~ms}$ & $11.8 \mathrm{~ms}$ \\
\hline
\end{tabular}

having one variable. Finally, JSNEAT is much faster and the results are more accurate as shown in Section VIII.

JSNaughty [21] formulates name recovery as a statistical machine translation from the minified code to the recovered code. First, due to the nature of ML, it faces the scalability issue in much higher time complexity. Second, JSNaughty uses a phrase-based translation model, which enforces a strict order between the recovered variable names in a function. This is too strict since a name of a variable might not need to occur before another name of another variable. Third, JSNaughty does not consider the task context of the variables. Finally, our training/testing time is much faster. In contrast, other deobfuscation methods use static/dynamic analyses [5], [13], [19].

Statistical NLP approaches have been used in SE. Naturalize [1] enforces a consistent naming style. Other applications of statistical NLP include code suggestion [9], [14], code convention [1], method name suggestion [2], API suggestions [18], code mining [3], type resolution [15], pattern mining [6]. Statistical NLP was used to generate code from text, e.g., SWIM [16], DeepAPI [7], Anycode [8], etc.

\section{CONClusion}

This paper presents JSNEAT, an IR-based approach to recover the variable names in minified JS code. We follow a data-driven approach by searching for names in a large corpus of open-source JS code. We use three types of contexts to match a variable in given minified code against the corpus. Our IR approach enables us to achieve high accuracy with less time complexity than the state-of-the-art approaches. JSNEAT achieves a high accuracy of $69.1 \%$ : the improvement of $66.1 \%$ and $43 \%$ over JSNice and JSNaughty, respectively. The time to recover for a file or for a variable with JSNEAT is twice as fast as with JSNice and $4 \mathrm{x}$ as fast as with JNaughty, respectively.

\section{ACKNOWLEDGMENT}

This work was supported in part by the US National Science Foundation (NSF) grants CCF-1723215, CCF-1723432, TWC1723198, CCF-1518897, and CNS-1513263. 


\section{REFERENCES}

[1] M. Allamanis, E. T. Barr, C. Bird, and C. Sutton. Learning natural coding conventions. In Proceedings of the International Symposium on Foundations of Software Engineering, FSE 2014, pages 281-293. ACM, 2014.

[2] M. Allamanis, E. T. Barr, C. Bird, and C. Sutton. Suggesting accurate method and class names. In Proceedings of the 10th Joint Meeting on Foundations of Software Engineering, ESEC/FSE 2015, pages 38-49. ACM, 2015.

[3] M. Allamanis and C. Sutton. Mining source code repositories at massive scale using language modeling. In Proceedings of the 10th IEEE Working Conference on Mining Software Repositories (MSR'13), pages 207-216. IEEE CS, 2013.

[4] D. M. Blei, A. Y. Ng, and M. I. Jordan. Latent dirichlet allocation. $J$. Mach. Learn. Res., 3:993-1022, Mar. 2003.

[5] M. Christodorescu and S. Jha. Static analysis of executables to detect malicious patterns. In Proceedings of the 12th Conference on USENIX Security Symposium - Volume 12, SSYM'03, pages 12-12. USENIX Association, 2003.

[6] J. M. Fowkes and C. A. Sutton. Parameter-free probabilistic API mining at github scale. CoRR, abs/1512.05558, 2015.

[7] X. Gu, H. Zhang, D. Zhang, and S. Kim. Deep API learning. In Proceedings of the 24th ACM SIGSOFT International Symposium on Foundations of Software Engineering, FSE 2016, pages 631-642. ACM, 2016.

[8] T. Gvero and V. Kuncak. Synthesizing Java expressions from freeform queries. In Proceedings of the 2015 ACM SIGPLAN International Conference on Object-Oriented Programming, Systems, Languages, and Applications, OOPSLA 2015, pages 416-432. ACM, 2015.

[9] A. Hindle, E. T. Barr, Z. Su, M. Gabel, and P. Devanbu. On the naturalness of software. In Proceedings of the 2012 International Conference on Software Engineering, ICSE 2012, pages 837-847. IEEE Press, 2012.

[10] JSNeat. https://mrstarrynight.github.io/JSNeat/.

[11] JSNice. https://files.sri.inf.ethz.ch/jsniceartifact/index.html.
[12] G. J. Klir and B. Yuan. Fuzzy sets and fuzzy logic: Theory and applicationss. Prentice-Hall, Inc., Upper Saddle River, NJ, USA, 1995.

[13] A. Moser, C. Kruegel, and E. Kirda. Exploring multiple execution paths for malware analysis. In Proceedings of the 2007 IEEE Symposium on Security and Privacy, SP '07, pages 231-245. IEEE Computer Society, 2007.

[14] L. Mou, G. Li, Z. Jin, L. Zhang, and T. Wang. TBCNN: A tree-based convolutional neural network for programming language processing. CoRR, abs/1409.5718, 2014.

[15] H. Phan, H. A. Nguyen, N. M. Tran, L. H. Truong, A. T. Nguyen, and T. N. Nguyen. Statistical learning of API fully qualified names in code snippets of online forums. In Proceedings of the 40th International Conference on Software Engineering, ICSE '18, pages 632-642. ACM, 2018.

[16] M. Raghothaman, Y. Wei, and Y. Hamadi. SWIM: Synthesizing what i mean: Code search and idiomatic snippet synthesis. In Proceedings of the 38th International Conference on Software Engineering, ICSE '16, pages 357-367. ACM, 2016.

[17] V. Raychev, M. Vechev, and A. Krause. Predicting program properties from "big code". In Proceedings of the 42nd Annual ACM SIGPLANSIGACT Symposium on Principles of Programming Languages, POPL '15, pages 111-124. ACM, 2015.

[18] V. Raychev, M. Vechev, and E. Yahav. Code completion with statistical language models. In Proceedings of the 35th ACM SIGPLAN Conference on Programming Language Design and Implementation, PLDI '14, pages 419-428. ACM, 2014.

[19] S. K. Udupa, S. K. Debray, and M. Madou. Deobfuscation: Reverse engineering obfuscated code. In Proceedings of the 12th Working Conference on Reverse Engineering (WCRE'05), pages 45-54. IEEE Computer Society, 2005.

[20] Uglify. https://github.com/mishoo/UglifyJS.

[21] B. Vasilescu, C. Casalnuovo, and P. Devanbu. Recovering clear, natural identifiers from obfuscated js names. In Proceedings of the 11th Joint Meeting on Foundations of Software Engineering, ESEC/FSE 2017, pages 683-693. ACM, 2017. 